\title{
Beurteilung der Arbeitsunfähigkeit - anspruchsvoller Routinevorgang
}

\section{Markus Baumgartner ${ }^{a}$, Roger Rudolph ${ }^{b}$}

a KD Dr. med., MAS MHC, Ärztlicher Direktor Clienia Schlössli AG und Chefarzt Psychiatriezentrum Wetzikon

b Prof. Dr. iur., Professur für Arbeits- und Privatrecht am Rechtswissenschaftlichen Institut der Universität Zürich

\section{Einleitung}

Es ist unbestritten, dass die Thematik der Arbeitsunfähigkeit in den vergangenen Jahren an Bedeutung gewonnen hat. Und, ob wir Ärzte es wollen oder nicht, bei der Beurteilung der Arbeitsunfähigkeit begeben wir uns unweigerlich in ein Spannungsfeld zwischen unserer rein ärztlichen Tätigkeit und den Erwartungen des Patienten, des Arbeitgebers und der Versicherungen. Dabei zeigt sich ein vermehrtes Misstrauen gegenüber Arbeitsunfähigkeitszeugnissen. Als Konsequenz davon müssen sich Ärzte vermehrt für die Qualität ihrer Zeugnisse verantworten, sei es gegenüber Vertrauens-

\section{L'essentiel en bref}

- La pratique montre que les certificats d'incapacité de travail plus détaillés (avec mention de la date de réévaluation, distinction entre composante horaire et de performance, etc.) sont nettement mieux acceptés par les employeurs et assureurs que les certificats courts.

- L'évaluation de l'incapacité de travail n'est pas seulement un acte suivi de possibles conséquences juridiques, mais surtout un acte thérapeutique avec un potentiel d'efficacité et d'effets secondaires.

- Les efforts en vue d'une intervention précoce et du maintien de l'emploi nécessitent un examen systématique des performances fonctionnelles au regard de la question "Qu'est-ce qui est encore possible?».

- Les explications de cet article se veulent un plaidoyer en faveur d'un changement de paradigme, en ce que nous adoptons une attitude fondamentalement positive en parlant de capacité de travail au lieu d'incapacité de travail. L'incapacité de travail se déduit alors indirectement de la capacité de travail.

- Ce n'est pas le diagnostic, mais la restriction fonctionnelle qu'il implique par rapport aux exigences de l'activité exercée jusque-là qui justifie l'incapacité de travail. Une évaluation fondée exige une connaissance de I'activité professionnelle concrète.

- L'insertion professionnelle nécessite une démarche coordonnée et la collaboration de toutes les parties prenantes (patients/salariés, médecins, employeurs, assureurs). La capacité de travail partielle devrait être privilégiée dans la mesure du possible, car elle facilite la réinsertion. ärzten, als Zeugen in einem arbeitsrechtlichen Zivilprozess oder gar als Angeschuldigte in einem Strafverfahren. Insbesondere ärztliche Zeugnisse von nur geringer inhaltlicher Aussagekraft (z.B. Arbeitsunfähigkeit von $100 \%$ bis auf weiteres) werden kritisch hinterfragt [1].

Doch nicht nur juristische Folgen sind zu bedenken, sondern vor allem die hohe Bedeutung der Dauer der Krankschreibung hinsichtlich Arbeitsplatzverlust und Berentung. Bereits nach drei Monaten wird die Rückkehr schwierig, nach einem Jahr Arbeitsunfähigkeit schaffen es nur noch wenige an den Arbeitsplatz zurück [2]. Somit haben Arbeitsintegrationsmassnahmen früh zu erfolgen. Auch im Kontext der Krankschreibung gilt der Grundsatz des Nicht-Schadens (cave: iatrogene Invalidisierung), d.h., Wirkungen und Nebenwirkungen von Arbeitsunfähigkeitszeugnissen sollen umsichtig bedacht werden.

Ferner sorgen AUF-Beurteilungen immer wieder für Verwirrung. Was meinen wir mit einer attestierten Arbeitsunfähigkeit von 50\%? Meinen wir damit eine Arbeitszeit von 50\% und eine Arbeitsleistung von 100\% oder eine Arbeitszeit von 100\% und eine Arbeitsleistung von 50\%? Bezieht sich dabei die Arbeitszeit auf ein Vollzeitpensum oder auf den effektiven Beschäftigungsgrad? Und wenn geklärt ist, worauf sich diese 50\% beziehen, ist der Arbeitnehmer nun fünf halbe Tage oder möglicherweise auch zweieinhalb Tage am Stück einsetzbar?

\section{Definition der Arbeitsunfähigkeit}

Definiert ist die Arbeitsunfähigkeit gemäss Art. 6 des Allgemeinen Teils des Sozialversicherungsrechts (ATSG) als «die durch eine Beeinträchtigung der körperlichen, geistigen oder psychischen Gesundheit bedingte, volle oder teilweise Unfähigkeit, im bisherigen Beruf oder Aufgabenbereich zumutbare Arbeit zu leisten. Bei langer Dauer wird auch die zumutbare Tätigkeit in einem anderen Beruf oder Aufgabenbereich berücksichtigt.» 


\section{Relevante Kriterien für die Beurteilung der Arbeitsunfähigkeit}

Versicherungsrechtlich relevant für die Beurteilung der Arbeitsunfähigkeit ist das Vorliegen einer Funktionseinschränkung in Bezug auf die bisher ausgeübte Tätigkeit. Das bedeutet gemäss den Leitlinien der Swiss Insurance Medicine, dass nicht die Diagnose im Vordergrund steht, sondern die Funktionseinschränkung (z.B. eine Aufmerksamkeitsstörung im Rahmen einer depressiven Episode), jedoch nicht isoliert, sondern eine Funktionseinschränkung in Bezug auf ein Anforderungsprofil, nämlich die Anforderungen der bisher ausgeübten Tätigkeit (z.B. als Buschauffeur). Nichtmedizinische Faktoren wie beispielsweise soziokulturelle Faktoren oder die Situation auf dem Arbeitsmarkt haben bei der Beurteilung keine Bedeutung zu spielen, da diese Risiken durch die Versicherungen nicht abgedeckt sind [3].

Letztlich soll die Beurteilung der Arbeitsunfähigkeit aufzeigen, dass das Ausüben der beruflichen Tätigkeit aus medizinischen Gründen nur teilweise möglich oder unmöglich ist, dem Therapieerfolg entgegenwirkt, einen Rückfall oder eine Verschlimmerung provozieren könnte oder andere Personen gefährden würde.

\section{Beurteilungsprozess}

Bei der Beurteilung der Arbeitsunfähigkeit gilt es der Gesundheit wie auch dem Arbeitsplatz des Patienten Sorge zu tragen. Erkrankte sollen so lange wie nötig und so kurz wie möglich dem Arbeitsplatz fernbleiben. Nach Möglichkeit gilt es eine teilweise Arbeitsfähigkeit anzustreben, denn auch eine begrenzte Präsenz am Arbeitsplatz erleichtert die Wiedereingliederung. Dabei empfiehlt es sich, ausgehend von der aktuellen Symptomatik nicht direkt auf die Arbeitsunfähigkeit zu schliessen, sondern primär auf die Arbeitsfähigkeit zu fokussieren, indem die beiden Komponenten der funktionellen Leistungsfähigkeit separat beurteilt werden. Das heisst, wir stellen uns die Fragen «Was geht noch in Bezug auf die Arbeitsleistung in Prozent bezogen auf die bisherige Leistung?» und «Was geht noch in Bezug auf die Arbeitszeit in Prozent bezogen auf den effektiven Beschäftigungsgrad?». Durch Multiplikation von Arbeitszeit in Prozent mit Arbeitsleistung in Prozent erhalten wir schliesslich die Arbeitsfähigkeit, und durch Subtraktion der Arbeitsfähigkeit von 100\% die Arbeitsunfähigkeit. Die Arbeitsunfähigkeit stellt quasi ein Nebenprodukt der Arbeitsfähigkeit dar, leitet sich also aus der Arbeitsfähigkeit ab [4].

Wichtig erscheint die Tatsache, dass sich die Beurteilung der Arbeitsfähigkeit immer auf die konkrete
Tätigkeit des Patienten bezieht. Somit können die Auswirkungen einer Funktionseinschränkung nur adäquat eingeschätzt werden, wenn wir Kenntnisse des jeweiligen Jobprofils haben. Dies setzt eine Arbeitsanamnese voraus.

Eine Hilfestellung zur differenzierten Beurteilung der funktionellen Leistungsfähigkeit bei psychischen Störungen bietet das Mini-ICF-APP, ein Fremdbeurteilungsinstrument zur Erfassung und Quantifizierung der Beeinträchtigungen und Ressourcen von Menschen mit psychischen Erkrankungen [5].

\section{Rechtliche Grundlagen}

Angesichts der grossen Bedeutung ärztlicher Zeugnisse wäre eigentlich zu erwarten, dass der Gesetzgeber diesem Befund durch eine zweckmässige gesetzliche Regelung Rechnung getragen hätte. Dem ist aber nicht so. Weder das Obligationenrecht (OR) noch das Arbeitsgesetz (ArG) regelt das ärztliche Zeugnis. Auch sonst finden sich in der schweizerischen Rechtsordnung keine Vorgaben zu Form und Inhalt ärztlicher Zeugnisse. Der Blick in das Gesetz hilft also nicht weiter, wenn Fragen nach den konkreten Modalitäten ärztlicher Zeugnisse zu beantworten sind.

Das Fehlen einschlägiger gesetzlicher Vorschriften heisst nun aber nicht, dass sich der Arzt in einem rechtsfreien Raum bewegt. Denn auch auf ihn sind die allgemeinen rechtlichen Regeln, namentlich des Zivilund des Strafrechts, anwendbar. Gegenüber dem Patienten steht der Arzt in einem vertraglichen Auftragsverhältnis (Art. 394ff. OR). Der Patient hat als Auftraggeber Anspruch darauf, dass der Arzt ihm eine Arbeitsunfähigkeit bescheinigt, wenn eine solche ausgewiesen ist. Umgekehrt muss und darf der Arzt keine Krankheit bestätigen, die in Tat und Wahrheit gar nicht besteht. Dies schon deshalb nicht, weil bei Ausstellen eines falschen ärztlichen Zeugnisses nach Artikel 318 des Strafgesetzbuchs (StGB) Freiheitsstrafe bis zu drei Jahren oder Geldstrafe droht. Die Bestimmung ist durchaus ernst zu nehmen, vor allem auch deshalb, weil nicht nur vorsätzliches, sondern auch fahrlässiges Handeln unter Strafe gestellt ist [6].

In einem weiteren Sinn ist bei der Erstellung von ärztlichen Zeugnissen auch die Verletzung des Berufsgeheimnisses (Art. $321 \mathrm{StGB}$ ) zu nennen. Die Ausstellung des Zeugnisses selbst stellt dabei eher selten ein Problem dar, da der Patient in der Regel selber darüber entscheidet, wem er das Zeugnis offenbaren will und wem nicht [7]. Für den Arzt heikler ist die nachgelagerte Phase, etwa wenn ein Arbeitgeber beim Arzt telefonisch nachfragt und weitere Auskünfte erhalten möchte, z.B. zur Diagnose oder zur Glaubwürdigkeit 


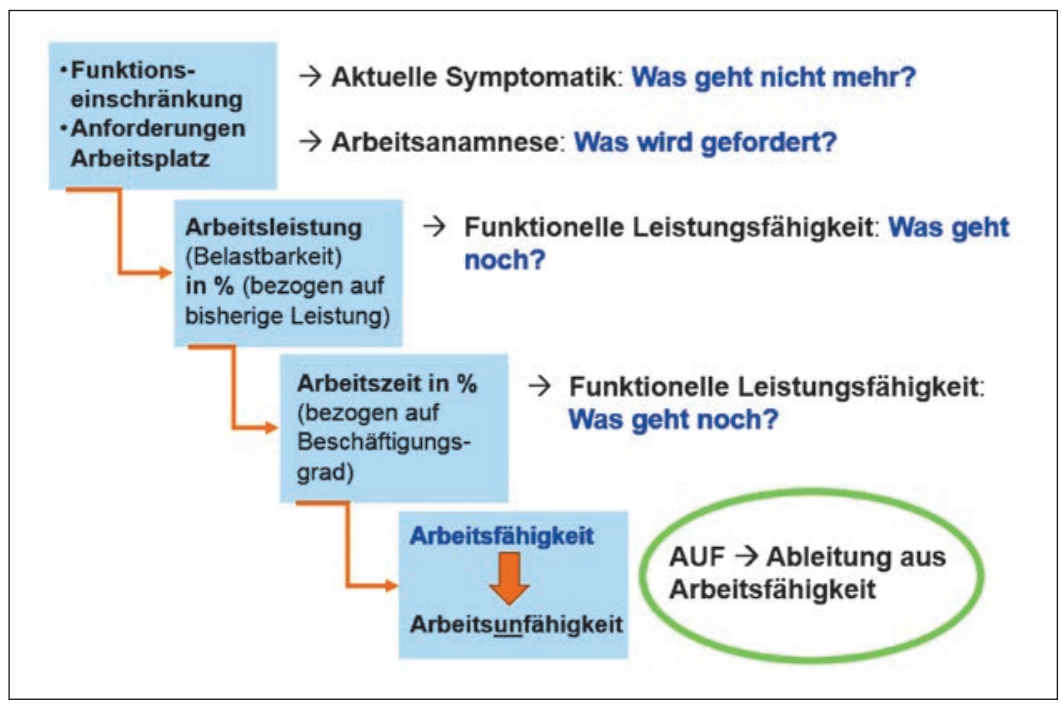

Berechnungsbeispiel: Ein Patient mit einem Vollzeitpensum kann während $50 \%$ der Arbeitszeit zu 50\% belastet werden. Daraus ergibt sich eine Arbeitsfähigkeit von 25\% $(50 \% \times 50 \%)$ bzw. eine Arbeitsunfähigkeit von 75\% (100-25\%).

der Patientenschilderung. Zu denken ist aber auch an den Vertrauensarzt des Arbeitgebers bzw. von dessen Krankentaggeldversicherung. Jeder Auskunft muss die Entbindung vom Berufsgeheimnis durch den Patienten vorausgehen.

Neben den eben erwähnten gesetzlichen Bestimmungen darf nicht vergessen werden, dass auch die Standesordnung FMH vom 12. Dezember 1996 in Artikel 34 einige wesentliche Grundsätze für die Erstellung ärztlicher Zeugnisse enthält: "Ärztliche Zeugnisse, Berichte und Gutachten sind Urkunden. Bei deren Ausstellung haben Arzt und Ärztin alle Sorgfalt anzuwenden und nach bestem Wissen ihre ärztliche Überzeugung auszudrücken. Der Zweck der Schriftstücke, das Ausstellungsdatum und ihre Empfänger sind anzugeben. Die Ausstellung von Gefälligkeitszeugnissen ist unzulässig.» Die Verletzung dieser Standesregeln kann mit standesrechtlichen Sanktionen bestraft werden, die vom Verweis bis zum Verbandsausschluss reichen (Art. 47 Standesordnung).

\section{Wichtige Aspekte beim Erstellen von AUF-Zeugnissen}

Obwohl konkrete gesetzliche Vorgaben fehlen, wie ein Arbeitsunfähigkeitszeugnis formuliert sein muss, hat sich in der Praxis ein gewisser Minimalstandard herausgebildet. Zum minimalen Zeugnisinhalt zählen Beginn und Grad sowie voraussichtliche Dauer der Arbeitsunfähigkeit. Aus versicherungsrechtlichen Gründen ist weiter zwischen Krankheit und Unfall zu unterscheiden. Ausserdem sollen die Adresskoordina- ten des Arztes ersichtlich sein (durch Stempel oder Briefpapier) und ist das Zeugnis zu datieren und zu unterzeichnen. Nach Art. 34 der Standesordnung FMH ist schliesslich der Adressat des Zeugnisses zu nennen, also in der Regel der Arbeitgeber [8].

Die praktische Erfahrung zeigt, dass es für den Patienten bzw. Arbeitnehmer, den Arbeitgeber, den Versicherer und letztlich auch den Arzt von Vorteil wäre, wenn das AUF-Zeugnis gegenüber dem Minimalinhalt einen etwas höheren Detaillierungsgrad aufweisen würde, weil ergänzende Informationen die Glaubwürdigkeit von Zeugnissen erheblich steigern können. Zu denken ist dabei an folgende Aspekte [9]:

- Dauer von Zeugnissen: Insbesondere zu Beginn einer möglicherweise längeren Arbeitsunfähigkeit soll die Dauer kurz gewählt werden und die Arbeitsunfähigkeit zeitnah neu beurteilt werden (z.B. 1 Woche nach Ausstellungsdatum). Die Nennung des Zeitpunkts der Neubeurteilung schafft Orientierung für Patient bzw. Arbeitnehmer sowie Arbeitgeber.

- Präzisieren, auf welches Pensum sich der Grad der Arbeitsunfähigkeit bezieht. Im Alltag erscheint es hilfreich, vom effektiven Beschäftigungsgrad auszugehen und dies auch explizit zu vermerken.

- Bei Teilarbeitsfähigkeit: Eine separate Beurteilung von Arbeitszeit (Präsenz) und Arbeitsleistung (Belastbarkeit) erhöht den Differenzierungsgrad. Gegebenenfalls Angabe, welche Arbeiten verrichtet bzw. derzeit nicht verrichtet werden können.

- Rückwirkende Zeugnisse: Arbeitsunfähigkeitszeugnisse werden grundsätzlich für die Zukunft, also prospektiv ausgestellt. Rückwirkende Zeugnisse sollen zurückhaltend ausgestellt werden und eine Woche nicht überschreiten [10]. Eine Rückdatierung ist nicht statthaft und kann den Tatbestand einer Urkundenfälschung oder eines falschen ärztlichen Zeugnisses erfüllen.

- Vermerk «Nach Angaben des Patienten»: Muss bei der Beurteilung auf nicht überprüfbare subjektive Angaben des Patienten abgestützt werden, soll das Zeugnis mit dem genannten Vermerk versehen sein.

- Bei arbeitsplatzbezogener Arbeitsunfähigkeit (z.B. Konflikte, Mobbing): Ist die Arbeitsunfähigkeit arbeitsplatzbezogen, sollte angegeben werden, ob und inwiefern der Patient in einem anderen Umfeld beim aktuellen Arbeitgeber oder ausserhalb beschäftigt werden kann (z.B. «100\% arbeitsunfähig im derzeitigen Job, jedoch voll einsetzbar in einer vergleichbaren Position in einem anderen Umfeld»).

- Es sollte auf eine sorgfältige Führung der Patientenakte geachtet werden. Sie kann für Ärzte zur «Le- 
Korrespondenz:

KD Dr. med.

Markus Baumgartner,

MAS MHC

Ärztlicher Direktor

Clienia Schlössli AG und

Chefarzt Psychiatrie-

zentrum Wetzikon

Schlösslistrasse 8

CH-8618 Oetwil am See

markus.baumgartner[at]

clienia.ch bensversicherung" werden, wenn sie jemals für ihre Zeugnisse vor Gericht oder in einem Strafverfahren Rechenschaft ablegen müssen.

Unabhängig von den genannten Aspekten ist dringend $\mathrm{zu}$ raten, Arbeitsunfähigkeitszeugnisse nur nach vorgängiger persönlicher Untersuchung auszustellen und bei der Übermittlung des Zeugnisses an den Arbeitgeber dieses vom Patienten überbringen zu lassen, weil spätestens dann dessen Einwilligung angenommen werden kann.

\section{Arbeitsintegration erfordert das Miteinander}

Es gibt Fälle, in denen der Arzt zum Schluss kommt, dass der Mitarbeiter seine angestammte Tätigkeit (vorübergehend) nur beschränkt oder nicht mehr ausüben kann. In diesem Fall ist das direkte Gespräch zwischen dem Arzt und dem Arbeitgeber empfeh- lenswert. Der Arzt hat dabei die Möglichkeit, Vorschläge oder Empfehlungen für die aktuelle oder eine künftig mögliche Tätigkeit des Mitarbeiters abzugeben. Die ärztliche Schweigepflicht darf dabei nicht als Vorwand zur Verweigerung der Zusammenarbeit zwischen der Ärzteschaft und den Arbeitgebern dienen. Da grundsätzlich aber nur der Patient (Arbeitnehmer) den Arzt von der Schweigepflicht entbinden kann, empfiehlt es sich, den Patienten für gemeinsame Gespräche mit dem Arbeitgeber zu motivieren und eine Entbindung von der Schweigepflicht einzuholen.

Als Grundlage für den Austausch und zur besseren Beurteilung der Arbeitsfähigkeit kann ein detailliertes Arbeitsunfähigkeitszeugnis auf der Basis einer vom Arbeitgeber verfassten Arbeitsplatzbeschreibung dienen.

\section{Bildnachweis}

Grafik: Markus Baumgartner

\section{Das Wichtigste in Kürze}

- Die Praxis zeigt, dass ausführlichere Arbeitsunfähigkeitszeugnisse (z.B. Nennung des Zeitpunkts der Neubeurteilung, Differenzierung zwischen Arbeitszeit und Arbeitsleistung) bei Arbeitgebern und Versicherern auf deutlich grössere Akzeptanz stossen als Kurzbescheinigungen.

- Die Beurteilung der Arbeitsunfähigkeit stellt nicht nur eine Handlung mit möglichen juristischen Folgen dar, sondern vor allem eine therapeutische Handlung mit Wirkungs- und Nebenwirkungspotenzial.

- Bestrebungen hinsichtlich Frühintervention und Arbeitsplatzerhalt bedingen eine konsequente Auseinandersetzung mit der funktionellen Leistungsfähigkeit geknüpft an die Frage "Was geht noch?».

- Die Ausführungen im vorliegenden Artikel wollen als Plädoyer für einen Paradigmenwechsel verstanden werden, indem wir im Sinne einer grundsätzlich positiven Haltung von Arbeitsfähigkeit anstatt von Arbeitsunfähigkeit sprechen. Die Arbeitsunfähigkeit leitet sich dann indirekt aus der Arbeitsfähigkeit ab.

- Nicht die Diagnose, sondern die durch sie bedingte Funktionseinschränkung in Bezug auf die Anforderungen der bisher ausgeübten Tätigkeit begründet die Arbeitsunfähigkeit. Eine fundierte Beurteilung setzt Kenntnisse der konkreten beruflichen Tätigkeit voraus.

- Arbeitsintegration erfordert ein koordiniertes Vorgehen und die Zusammenarbeit aller Beteiligten (Patienten/Arbeitnehmer, Ärzte, Arbeitgeber, Versicherer). Nach Möglichkeit ist eine teilweise Arbeitsfähigkeit anzustreben, weil dadurch die Wiedereingliederung erleichtert wird.
Literatur

1 Rudolph R. Arbeitsunfähigkeitszeugnisse: Ärzte zunehmend im Fokus der Justiz (Teil 1). Schweiz Ärzteztg. 2010;91(22):864-7.

2 Fit Mind, Fit Job: From Evidence to Practice in Mental Health and Work. OECD Publishing, 2015.

http://www.oecd.org/els/fit-mind-fit-job-9789264228283-en.htm

3 Arbeitsfähigkeit: Leitlinie zur Beurteilung der Arbeitsunfähigkeit nach Unfall und Krankheit, 4. Auflage. Swiss Insurance Medicine, 2013.

https://www.swiss-insurance-medicine.ch/tl files/firstTheme/ PDF\%2ODateien\%2Oab\%2O2015/4\% 2OFachwissen\%20nachschlagen/Arbeitsunfaehigkeit\%20(Zeugnisse\%20verlinkt\%20mit\%20 medforms.ch)/SIM\%2OAUF\%2OBroschuere_2013 D.pdf

4 Meyer-Blaser. Der Rechtsbegriff der Arbeitsunfähigkeit und seine Bedeutung in der Sozialversicherung. In: Schaffhauser/Schlauri. Schmerz und Arbeitsunfähigkeit. St. Gallen 2003. S. 47f.

5 Linden, Baron et Muschalla. Mini-ICF-APP - Mini-ICF-Rating für Aktivitäts- und Partizipationsbeeinträchtigungen bei psychischen Erkrankungen. Hogrefe-Verlag, 2. Auflage, 2015

6 Dürr Brunner C. Strafrechtliche Relevanz von Medizinischen Berichten. Schweiz Ärzteztg. 2009;90(23):920-2.

7 Anders aber dann, wenn der Arzt das Zeugnis direkt dem Arbeitgeber zustellt und er darin ohne Zustimmung des Patienten z.B. die Diagnose offenbart.

8 Art. 34 der Standesordnung der FMH verlangt Letzteres ausdrücklich.

9 Rudolph R. Arbeitsunfähigkeitszeugnisse: Ärzte zunehmend im Fokus der Justiz (Teil 2). Schweiz Ärzteztg. 2010;91(23):912-5.

10 Empfehlungen der Ärztegesellschaft Zürich (AGZ) zum Ausstellen von Ärztlichen Zeugnissen, abrufbar über die Website der AGZ: https://aerzte-zh.ch/pdf/Informationen/merkblaetter/Empf\%20 Ae\%20Zeugnisse\%20MIT\%20 LOGO_NEU.pdf 